\title{
ALTERAÇÕES DAS FUNÇÕES EXECUTIVAS EM CRIANÇAS E ADOLESCENTES
}

\author{
Natália Martins Dias \\ Psicóloga, Mestre e Doutoranda do Programa de Pós-Graduação em Distúrbios do \\ Desenvolvimento, \\ Universidade Presbiteriana Mackenzie - Bolsista FAPESP
}

\begin{abstract}
Amanda Menezes
Psicóloga, Mestre em Avaliação Psicológica e Doutoranda do Programa de Pós-Graduação em Distúrbios do Desenvolvimento, Universidade Presbiteriana Mackenzie - Bolsista Mérito MackPesquisa
\end{abstract}

\begin{abstract}
Alessandra Gotuzo Seabra
Doutora e Pós-Doutora em Psicologia pela Universidade de São Paulo Docente do Programa de Pós-Graduação em Distúrbios do Desenvolvimento, Universidade Presbiteriana Mackenzie - Bolsista de Produtividade do CNPq
\end{abstract}

\begin{abstract}
Resumo
Funções executivas constituem um conjunto de processos cognitivos que permitem a regulação da cognição e do comportamento, possibilitando o engajamento do indivíduo em ações complexas. Essas habilidades estão comprometidas em uma diversidade de condições clínicas e este artigo teórico teve como objetivo oferecer uma revisão não-sistemática de alguns quadros nos quais estas habilidades apresentam-se prejudicadas, sobretudo em crianças e adolescentes. Verificou-se que condições como transtorno do déficit de atenção e hiperatividade, transtorno obsessivo-compulsivo, síndrome de Tourette, transtornos globais do desenvolvimento, transtornos de aprendizagem como a dislexia e a discalculia, além de alterações cromossômicas, como a síndrome de Down e de Prader-Willi, cursam com algum grau de comprometimento do funcionamento executivo. Este conhecimento tem implicações à prática e à pesquisa psicológica e pode ser útil a profissionais que atuam com crianças e adolescentes com alguma destas condições, contribuindo a um maior conhecimento acerca das funções executivas e suas alterações.
\end{abstract}

Palavras-chave: funcionamento executivo, neuropsicologia, desenvolvimento.

\section{EXECUTIVE FUNCTIONS DAMAGE IN CHILDREN AND ADOLESCENTS}

\begin{abstract}
Executive functions are a set of cognitive skills that allows the cognition and the behavior regulation, enabling an individual to take part in complex actions. Those abilities are committed in a diversity of clinical conditions and this theoretical article aimed offer an unsystematic revision of some conditions in which these abilities are injured, especially in children and adolescents. It was verified that conditions as Attention Deficit Hyperactivity Disorder, ObsessiveCompulsive Disorder, Tourette Syndrome, Pervasive Developmental Disorders, Learning Disabilities, like as Dyslexia and Dyscalulia, beyond cromossomic alterations, as Down's and Prader-Willi Syndromes, follow with some degree of executive functioning compromise. This knowledge has implications to the psychological practice and research, and can be useful to professionals who act with children and adolescents with some of these conditions, contributing to a greater knowledge about executive functions and its damages.
\end{abstract}

Keywords: executive functioning, neuropsychology, development. 


\title{
ALTERACIONES DE LAS FUNCIONES EJECUTIVAS EN NIÑOS Y ADOLESCENTES
}

\begin{abstract}
Resumen
Las funciones ejecutivas son un conjunto de procesos cognitivos que permiten la regulación de la cognición y del comportamiento, lo que permite al individuo a realizar acciones complejas. Estas habilidades están comprometidas en una variedad de condiciones clínicas y este artículo teórico tiene como objetivo ofrecer una revisión no sistemática de algunas condiciones que tienen estas habilidades perjudicadas, especialmente en niños y adolescentes. Se constató que los problemas como el trastorno por déficit de atención e hiperactividad, el trastorno obsesivo-compulsivo, síndrome de Tourette, trastornos generalizados del desarrollo, trastornos del aprendizaje como la dislexia y discalculia, y alteraciones cromosómicas como síndrome de Down y síndrome de Prader-Willi, se producen con algún grado de daño en el funcionamiento ejecutivo. Este conocimiento tiene implicaciones para la práctica y la investigación psicológica, y puede ser útil a los profesionales que trabajan con niños y adolescentes con alguna de estas condiciones, contribuyendo a un mayor conocimiento de las funciones ejecutivas y sus alteraciones.
\end{abstract}

Palavras clave: funcionamiento ejecutivo, neuropsicología, desenvolvimiento.

\section{INTRODUÇÃO}

As funções executivas são um conjunto de habilidades cognitivas que permitem ao sujeito engajar-se em comportamentos orientados a objetivos, realizando ações voluntárias, independentes, auto-organizadas e direcionadas a metas. Estas habilidades são especialmente importantes diante de situações novas ou em circunstâncias que exigem ajustamento, adaptação ou flexibilidade do comportamento para as demandas do ambiente (Elliott, 2003; Gazzaniga, Ivry \& Mangun, 2006; Huizinga, Dolan \& Molen, 2006). Segundo Lezak, Howieson e Loring (2004), tais funções são fundamentais ao direcionamento e regulação de várias habilidades intelectuais, emocionais e sociais.

Estas habilidades de alta ordem estão diretamente relacionadas ao córtex pré-frontal (Gazzaniga et al, 2006). Tal estrutura, aquisição filogênica dos mamíferos, encontra-se mais desenvolvida na espécie humana do que em qualquer outra (Estévez-González, García-Sánchez \& Barraquer-Bordas, 2000; Gil, 2002). Goldberg (2002) enfatiza que nenhuma outra perda cognitiva pode ser tão comprometedora para o comportamento humano quanto a das funções executivas. Segundo o autor, déficits executivos podem estar relacionados a diferentes condições, como alguns transtornos psiquiátricos, demências, lesões traumáticas, entre outros.

Atualmente, há na literatura relativo consenso de que estas habilidades constituem um construto multidimensional. Ou seja, os processos componentes 
das funções executivas caracterizam operações distintas, ainda que relacionadas (Miyake, Friedman, Emerson, Witzki \& Howerter, 2000). Essa visão multifacetada é corroborada por pesquisas realizadas com baterias de avaliação neuropsicológica e técnicas de neuroimagem, as quais têm sugerido evidências da existência de diferentes aspectos relacionados ao córtex pré-frontal e às funções executivas (Dias, 2009; Elliot, 2003; Huizinga et al, 2006; Menezes, 2008; Miyake et al, 2000). Araújo (2004) relata, por exemplo, que lesões em diferentes partes dos lobos frontais produzem síndromes clinicamente diferentes, o que corrobora a idéia de diversidade funcional e complexidade da região.

Corroborando tal visão, considera-se que as funções executivas envolvam diferentes processamentos cognitivos, como a atenção seletiva, integração e manipulação das informações relevantes na memória de trabalho, controle de impulsos, planejamento, intenção, efetivação das ações, flexibilidade cognitiva e comportamental, e monitoramento das atitudes (Duncan, Johnson, Swales \& Frees, 1997; Fuster, 1997; Gazzaniga et al, 2006; Lezak et al, 2004; Pliszka, 2004; Sternberg, 2008). De forma conjunta, esses processos cognitivos agrupados sob o termo 'funções executivas' permitem a um indivíduo iniciar, planejar, seqüenciar e monitorar seus comportamentos e cognições (Gazzaniga et al, 2006; Pliszka, 2004).

Este artigo teórico tem como objetivo oferecer uma revisão nãosistemática, ilustrando alguns quadros nos quais há relatos na literatura de comprometimentos nas funções executivas. Este conhecimento é útil a profissionais que atuam, sobretudo nos contextos clínico e escolar, com crianças e adolescentes.

\section{As funções executivas e suas alterações}

O comprometimento das habilidades executivas, caracterizando a chamada síndrome disexecutiva, pode compreender alterações cognitivo-comportamentais diversas, associadas ao prejuízo de seus processos componentes, tais como dificuldades na seleção de informação, distratibilidade, dificuldades na tomada de decisão, problemas de organização, comportamento perseverante ou estereotipado, dificuldade no estabelecimento de novos repertórios comportamentais, dificuldades de abstração e de antecipação das conseqüências de seu comportamento, impondo uma série de problemas à vida diária (Muñoz- 
Céspedes \& Tirapu-Ustárroz, 2004; Strauss, Sherman \& Spreen, 2006). A essas dificuldades, Lent (2001) acrescenta o imediatismo comportamental e o prejuízo no ajuste social do comportamento, e Saboya, Franco e Mattos (2002) destacam os prejuízos em habilidades de planejamento, memória evocativa e mesmo em linguagem expressiva. Em suma, alterações estruturais ou funcionais dos lobos pré-frontais ou de seus circuitos podem ocasionar diversos transtornos comportamentais desadaptativos (García-Molina, 2008).

Em situações de testagem, indivíduos com disfunções executivas tendem a apresentar pobre iniciação, problemas de planejamento e organização da tarefa, dificuldades na inibição e na mudança de respostas, na geração e implementação de estratégias, assim como pobre memória de trabalho e ocorrência freqüente de erros perseverativos. Mais especificamente, pacientes com lesão pré-frontal apresentam prejuízo em uma variedade de medidas, como no Teste de Classificação de Cartas de Wisconsin, Teste de Stroop e testes de fluência (Strauss et al, 2006).

Todos esses apontamentos são bem exemplificados no estudo de Rojas, Tapia, Yañez e De La Cruz (2008). Os autores avaliaram um menino de oito anos com agenesia da região pré-frontal do lobo frontal. Para além de prejuízos em testes formais do funcionamento executivo, os autores descreveram a presença de problemas comportamentais, como a dificuldade de julgar ações, a impossibilidade de internalizar regras sociais e ausência de limites, além de problemas motivacionais para encerrar atividades iniciadas, mesmo aquelas de cunho recreativo.

De fato, alterações no controle executivo já têm sido relatadas em uma diversidade de casos, especificamente em crianças e adolescentes, como nos transtornos de comportamento disruptivo, tal como o Transtorno do Déficit de Atenção e Hiperatividade - TDAH (Alfano, 2005; Assef, 2005; Coutinho et al, 2007; Nigg, 2001; Wolfe, 2004); transtornos invasivos ou globais do desenvolvimento, a exemplo daqueles compreendidos no espectro autista (Landa \& Goldberg, 2005; Orsati, Schwartzman, Brunoni, Mecca \& Macedo, 2008; Steele, Minshew, Luna \& Sweeney, 2007); transtornos de aprendizagem, como a dislexia (Beneventi, Tønnessen, Ersland \& Hugdahl, 2010; Reiter, Tucha \& Lange, 2005; Salgado, Lima \& Ciasca, 2008) e a discalculia (Argollo, 2008; Balbi, 2008; McLean \& Hitch, 1999); quadros neurológicos, como a epilepsia (Levav, 2008); e também naqueles caracterizados por alterações cromossômicas, como a 
Síndrome de Down (Lanfranchi, Jerman, Dal Pont, Alberti \& Vianello, 2010) e a Síndrome de Prader-Willi (Jauregi et al, 2007). Para além de quadros clínicos bem definidos, relações também tem sido estabelecidas entre as habilidades executivas e o desempenho escolar (Capovilla \& Dias, 2008; Gathercole, Alloway, Willis \& Adams, 2006).

E não somente nos quadros supracitados as alterações executivas se fazem presentes. Em uma revisão acerca dos transtornos do funcionamento executivo, Papazian e colaboradores (2006) concluíram que alterações em tais funções foram relatadas também em crianças com quadros diversos, como traumatismo cerebral fechado, Síndrome de Tourette e Transtorno ObsessivoCompulsivo (TOC). Mahone e Silverman (2008) apontam que a disfunção executiva é frequentemente mais severa na presença da tríade de comorbidades entre TDAH, Síndrome de Tourette e TOC. Além, em estudos longitudinais de seguimento, tem sido também relatados prejuízos executivos, sobretudo déficits de flexibilidade cognitiva e planejamento, em crianças prematuras de baixo peso ao nascimento (Cuevas \& Waisburg, 2008).

Apesar dos diversos quadros nos quais alterações do funcionamento executivo foram relatadas, grande número de evidências está disponível discorrendo sobre a disfunção executiva no TDAH. Em artigo de revisão, Rebollo e Montiel (2006) consideram e resgatam a proposta de Barkley (1997), aludindo que o TDAH reflete uma alteração no funcionamento executivo, mais especificamente, um prejuízo na habilidade de inibir o comportamento, o que teria conseqüências diretas sobre o auto-controle do indivíduo. Nigg (2001) também partiu desta premissa e focou uma série de estudos que avaliaram o controle inibitório em crianças e adolescentes com TDAH, considerando somente o tipo combinado, com o objetivo principal de verificar evidências de um déficit primário na habilidade de inibição subjacente ao transtorno. De modo geral, o autor concluiu que a aceitação de um déficit no controle inibitório como central ou causal ao TDAH carece ainda de comprovação, apesar de sua freqüente associação com o transtorno. Alguns dados interessantes do estudo mostraram que indivíduos com TDAH tipo combinado apresentam um déficit pronunciado em tarefas de inibição motora, enquanto alguns dados apontam para déficits menos expressivos no controle de interferência e resultados ainda inconclusivos com relação às tarefas de inibição cognitiva. O autor levantou a hipótese de que talvez os déficits executivos no TDAH tipo desatento estariam mais relacionados 
a dificuldades de inibição cognitiva do que à supressão de uma resposta motora, por exemplo. Estudos futuros devem endereçar esta questão.

As conclusões da revisão de Mahone e Silverman (2008) dão algum suporte às de Nigg (2001). Estes pesquisadores verificaram que a maturação do córtex pré-frontal de crianças com TDAH é atrasada de dois a cinco anos em relação a controles, porém a maturação das áreas motoras primárias destas crianças é adiantada. Ou seja, estes achados sugerem um desenvolvimento neurológico atípico que conduziria a uma excessiva atividade motora destas crianças e uma falha em inibir tais comportamentos inadequados pelo córtex pré-frontal, ainda imaturo. Também prejuízos atencionais foram relatados em uma amostra não clínica de crianças e adolescentes com sintomas de TDAH (Coutinho et al, 2007).

Como se pode observar, déficits no funcionamento executivo estão frequentemente associados ao diagnóstico de TDAH e podem conduzir a comprometimentos em diversas atividades cotidianas; em crianças, sobretudo na escola. Por exemplo, prejuízos na memória de trabalho muito frequentemente podem incorrer em dificuldades na compreensão de leitura, mesmo na presença de boas habilidades de reconhecimento de palavras (Mahone \& Silverman, 2008). De fato, postergar tarefas e/ou não finalizá-las, dificuldade em focalizar e sustentar a atenção, dificuldade em organização e hierarquização, deficiência em memória de trabalho e prospectiva, o que pode ocasionar o esquecimento de datas e compromissos, são alterações executivas frequentemente relatadas em associação com o transtorno e que levam a significativo impacto no desempenho funcional do indivíduo (Saboya, Saraiva, Palmini, Lima \& Coutinho, 2007). Perante estas dificuldades, para estes autores o TDAH deve ser compreendido como uma síndrome disexecutiva.

Algumas evidências de alterações executivas em crianças com Distúrbio Específico de Linguagem (DEL) também foram encontradas, sugerindo prejuízos em habilidades de atenção, memória, inibição e memória de trabalho associadas ao diagnóstico (Im-Bolter, Johnson \& Pascual-Leone, 2006). Outras investigações também têm sugerido comprometimento destas habilidades nos quadros de transtorno de aprendizagem, especificamente, a dislexia (Beneventi et al, 2010; Reiter et al, 2005; Salgado et al, 2008) e a discalculia (Argollo, 2008; Balbi, 2008; McLean \& Hitch, 1999). 
Por exemplo, Reiter e colaboradores (2005) investigaram diversos aspectos das funções executivas em crianças com diagnóstico de dislexia. Seus dados mostraram que as crianças com o transtorno de leitura apresentavam desempenho inferior ao do grupo controle em tarefas de memória de trabalho, fluência verbal e de figuras, e controle inibitório, quando avaliado por tarefas complexas. Já a avaliação da resolução de problemas mostrou-se parcialmente comprometida, enquanto a habilidade de formação de conceitos não apresentou comprometimento. Beneventi e colaboradores (2010), por sua vez, buscaram averiguar a relação entre a dislexia e uma das habilidades executivas, a memória de trabalho. Os dados permitiram identificar que o grupo com dislexia apresentou, em relação ao grupo controle, reduzida ativação nos córtices préfrontal e parietal, além do cerebelo. Seus resultados também indicaram que os disléxicos apresentavam comprometimento na memória de trabalho. No âmbito nacional, também Salgado e colaboradores (2008) encontraram evidências de prejuízo em algumas habilidades executivas em crianças com dislexia.

Porém, conforme apontam Dias, Trevisan, Menezes, Godoy e Seabra (no prelo), não é claro qual papel estes comprometimentos executivos podem ter na manifestação dos principais sintomas da dislexia. As autoras ainda hipotetizam que seria plausível que as alterações executivas, como por exemplo o comprometimento na memória de trabalho, prejudicassem a compreensão de leitura, tendo menor efeito, se algum, sobre os processos de decodificação. Algumas evidências nesta direção são oferecidas pelo estudo de Cutting, Materek, Cole, Levine e Mahone (2009). Os autores investigaram os processos neuropsicológicos associados a diferentes tipos de dificuldades de leitura, ou seja, dificuldades na leitura de palavras isoladas e déficit na compreensão de leitura. Apesar de crianças com ambas as dificuldades apresentarem prejuízo em fluência de leitura e habilidades de linguagem oral em relação aos controles, as crianças e adolescentes com déficits específicos de compreensão mostraram, tanto em relação aos controles quanto aos pares com dificuldades na leitura de palavras isoladas, prejuízo em funções executivas, especificamente na habilidade de planejamento e organização.

Evidências apontam ainda para um comprometimento das habilidades executivas na discalculia ou Transtorno da Matemática (conforme APA, 2002). Por exemplo, McLean e Hitch (1999) verificaram prejuízo executivo e, de modo mais específico, no subsistema visoespacial da memória de trabalho em crianças 
com o transtorno. Também Balbi (2008), a partir de dois relatos de caso com crianças de sete e oito anos com o mesmo diagnóstico, relatou alterações na memória de trabalho associados ao quadro, bem como prejuízos no desempenho atencional. No âmbito nacional, Argollo (2008) realizou estudo com a bateria Nepsy II e observou que, apesar de habilidades de linguagem, função sensóriomotora e de percepção social preservadas, crianças com discalculia apresentaram prejuízo no processamento visuoespacial, memória visual e em atenção e funções executivas, quando comparadas a controles sem o transtorno de aprendizagem.

Também nos transtornos globais ou invasivos do desenvolvimento (TGD conforme OMS, 1993; TID conforme APA, 2002) há relatos de alterações no funcionamento executivo. Estudos tem provido tais evidências utilizando distintas formas de mensuração. Por exemplo, Orsati e colaboradores (2008) utilizaram técnica de rastreamento de movimento ocular para avaliar habilidades executivas com recurso às tarefas de Sacada preditiva e Anti-sacada. Verificaram que os participantes com TGD apresentaram maior dificuldade em ambas as tarefas, ou seja, prejuízo na regulação da atenção e na preparação da resposta (sacada preditiva), tanto quanto em inibir e controlar movimentos reflexos e respostas preponderantes (anti-sacada) em relação aos controles. Ambas as medidas se mostraram positiva e moderadamente relacionadas com a idade, ou seja, com o aumento da idade há uma melhora na regulação da atenção e preparação para resposta requerida, tanto quanto na inibição da resposta preponderante. Os autores postularam que as diferenças na execução e inibição dos movimentos oculares na população com TGD poderiam estar relacionadas à forma como estes indivíduos extraem informação do meio e esta, por sua vez, pode ter repercussões em como o indivíduo opera sobre este meio. Esta suposição faz perceber a importância das habilidades executivas no TGD.

Este estudo corrobora outros, conduzidos com amostras mais específicas. Em 2005, Landa e Goldberg encontraram evidência de prejuízo em memória de trabalho espacial e planejamento em 19 crianças e adolescentes autistas de alto funcionamento. Os autores observaram nos participantes autistas erros por perseveração e pobres estratégias empregadas para a resolução das tarefas; além, encontraram correlação positiva e significativa entre a medida de memória de trabalho e uma de funcionamento social, conforme mensurado por protocolo de observação (Landa \& Goldberg, 2005). Também Steele e colaboradores 
(2007) encontraram prejuízo de memória de trabalho em 29 adolescentes e adultos autistas de alto funcionamento em relação a controles de desenvolvimento típico, pareados em idade e QI. Tal dificuldade se tornava mais evidente progressivamente com o aumento da demanda da tarefa.

De modo geral, os resultados de pesquisas tem sido consistentes em apontar prejuízo no funcionamento executivo em indivíduos com TGD. Tal fato levou à suposição de que um déficit em tais funções poderia ser primário ao distúrbio, conforme apontam Yerys, Hepburn, Pennington e Rogers (2007). Foi com o objetivo de verificar esta hipótese que estes autores delinearam seu estudo. Mais especificamente, eles objetivaram verificar se os déficits executivos estariam já presentes em crianças autistas antes dos três anos de idade e determinar se tais déficits seriam secundários ao transtorno ou se deveriam ser referidos como fatores de risco cognitivo para o distúrbio. Foram tomadas medidas de memória de trabalho, inibição e flexibilidade, porém verificou-se que as crianças autistas até três anos de idade não demonstraram déficits em comparação aos seus controles, tanto os pareados por idade mental quanto cronológica. O desempenho similar em idades precoces seria consistente com um déficit secundário das funções executivas no autismo e é condizente com pesquisas realizadas com pré-escolares; porém não com outras, conduzidas com adultos e crianças em idade escolar. Os autores concluem que o prejuízo executivo parece não ser primário ou causal ao autismo e que tais déficits aumentam com a progressão da idade nesta população (Yerys et al, 2007). Porém, apesar de não comentado pelos autores, é possível que estes resultados tenham sido encontrados em função de dificuldades inerentes à avaliação de crianças nesta faixa etária, entre elas, cabe citar a possibilidade de uso de testes pouco sensíveis e psicometricamente inadequados à avaliação de amostras tão jovens.

Alterações nas funções executivas foram também descritas na Síndrome de Prader-Willi (Jauregi et al, 2007) e na Síndrome de Down (Lanfranchi et al, 2010; Rowe et al, 2006). Na primeira, os autores tinham como objetivo identificar os processos prejudicados, o que poderia auxiliar na compreensão dos mecanismos subjacentes ao déficit de cognição social e desordem de comportamento social frequentemente associados à síndrome. Encontraram que seus participantes com Prader-Willi, entre adolescentes e adultos, demonstraram déficit atencional e em habilidades executivas, com dificuldades de organização e 
planejamento. Os autores argúem que alguns resultados podem ser específicos da síndrome de Prader-Willi, mas podem também refletir a deficiência intelectual. Porém, seus achados também sustentaram que um melhor desenvolvimento cognitivo não caracteriza fator de proteção contra os déficits neuropsicológicos evidenciados. Ressaltam a importância das funções executivas no comportamento e funcionamento adaptativo, de modo que a avaliação e o delineamento do perfil cognitivo de cada síndrome são relevantes e podem ampliar o conhecimento atual de cada quadro, possibilitando seu melhor manejo (Jauregi et al, 2007).

Já o estudo de Rowe e colaboradores (2006) examinou aspectos do funcionamento executivo em indivíduos com Síndrome de Down em comparação a controles com diagnóstico de Transtorno de aprendizagem e, apesar de seu estudo ter sido conduzido com adultos, prove informação útil ao entendimento das alterações executivas associadas a esta condição. Os grupos foram pareados em função de uma medida de vocabulário. Verificou-se que, mesmo em comparação com um grupo controle que também apresentava comprometimentos, a defasagem no funcionamento executivo dos participantes sindrômicos foi evidente, sobretudo em medidas de flexibilidade e atenção sustentada. Os autores concluíram que o déficit nas funções executivas é uma característica da Síndrome de Down, possível conseqüência de um prejuízo no desenvolvimento do córtex pré-frontal, porém não discutiram se estas diferenças podem ser devidas à deficiência intelectual, e outros estudos devem investigar de modo mais controlado tal questão. A avaliação neuropsicológica e, sobretudo, das funções executivas e funções de memória é de extrema importância na Síndrome de Down, haja a alta incidência de demências em idade precoce nesta população.

Um estudo recente corroborou estes achados em uma amostra de adolescentes com a síndrome (Lanfranchi et al, 2010). Os autores aplicaram a seus participantes, adolescentes com Síndrome de Down e crianças de desenvolvimento típico pareadas por idade mental, uma vasta bateria de avaliação neuropsicológica. Os resultados evidenciaram comprometimento executivo nos adolescentes sindrômicos, com prejuízos, sobretudo, nas tarefas de flexibilidade, planejamento e resolução de problemas, memória de trabalho e inibição. 


\section{CONSIDERAÇÕES FINAIS}

Este artigo teve o objetivo de apresentar algumas condições nas quais as distintas habilidades envolvidas nas funções executivas encontram-se comprometidas. Não caracteriza uma revisão sistemática e tampouco pretende esgotar o tema. Mas sumaria um razoável corpo de estudos que revelaram alterações nas funções executivas em quadros bastante diversos como TDAH ou TOC e algumas síndromes genéticas.

Compreender estas alterações faz-se importante e tem conseqüências diretas à prática e à pesquisa em psicologia e neuropsicologia. Dentre as implicações práticas pode-se mencionar, como ilustração, aquelas de cunho psicoeducacional. Por exemplo, em contexto de sala de aula, com um aluno com diagnóstico de transtorno de aprendizagem e de posse do conhecimento de que déficits de memória de trabalho estão associados a esse diagnóstico, um professor poderia minimizar as demandas das tarefas propostas sobre esta habilidade. Para isso, poderia propor tarefas mais estruturadas, oferecer instruções breves e objetivas, retomar com maior freqüência os objetivos da tarefa, sendo auxiliado neste processo pelo psicólogo escolar. No âmbito clínico, seria pertinente a atuação para estimular o desenvolvimento destas habilidades ou suplantar seu comprometimento com suportes externos.

As possíveis implicações à pesquisa, por sua vez, remetem essa discussão a dois pontos principais. Primeiramente, é fundamental dispor de instrumentos psicometricamente adequados à avaliação das habilidades executivas em crianças e adolescentes e, outro ponto de destaque, é igualmente importante mapear as tendências desenvolvimentais de cada uma destas habilidades ao longo de uma ampla faixa etária, pois, somente conhecendo o desenvolvimento normal destas habilidades será possível compreender mais profundamente as alterações e desvios em relação a este. Tais estudos ainda são escassos no Brasil, mas algumas investigações já tem se direcionado a estas questões, a exemplo das contribuições de Argollo e colaboradores (2009), Dias (2009), Menezes (2008) e Natale, Teodoro, Barreto e Haase (2008).

Por fim, espera-se que este artigo possa ser uma ferramenta informativa ao alcance de clínicos e educadores, assim como de outros profissionais que atuam com crianças e adolescentes que apresentem os diversos quadros sumariados. Desta forma, poderá ser útil a um maior conhecimento acerca das funções executivas e de suas alterações. 


\section{REFERÊNCIAS}

Alfano, A. (2005). Avaliação neuropsicológica no transtorno do déficit de atenção e hiperatividade (TDAH): contribuições para uma intervenção eficaz. In L.E.L.R. Valle (Org.), Neuropsicologia e Aprendizagem: para viver melhor (pp.89-94). Ribeirão Preto, SP: Tecmedd e SBNp.

APA - Associação Psiquiátrica Americana. (2002). Manual Diagnóstico e Estatístico de Transtornos Mentais DSM-IV-TR tm (4a ed.). Porto Alegre, RS: Artmed.

Araújo, C. (2004). Avaliação neuropsicológica das disfunções executivas. Em: Sociedade Brasileira de Neuropsicologia. (2004). Temas multidisciplinares de neuropsicologia e aprendizagem (pp. 209-216). São Paulo: Robe.

Argollo, N. (2008). Avaliação Neuropsicológica da discalculia do desenvolvimento com o Nepsy II: avaliação neuropsicológica do desenvolvimento. In A. Sennyey, F. C. Capovilla \& J. M. Montiel (Orgs.), Transtornos de Aprendizagem: da avaliação à reabilitação (pp. 115-124). SP: Artes Médicas.

Argollo, N., Bueno, O., Shaver, B., Godinho, K., Abreu, K., Durán, P., et al (2009). Adaptação transcultural da bateria NEPSY - Avaliação neuropsicológica do desenvolvimento: Estudo-piloto. Avaliação Psicológica, $8(1), 59-75$.

Assef, E. C. (2005). Avaliação das funções executivas em crianças com Transtorno de Déficit de Atenção e Hiperatividade. Dissertação de mestrado, Programa de Pós-Graduação Stricto Sensu em Psicologia, Universidade São Francisco, Itatiba, São Paulo.

Balbi, A. (2008). Two clinical cases of children with arithmetic learning disability. Journal of the International Neuropsychological Society, 14(Suppl. 2), 3737.

Barkley, R. A. (1997). Behavioral inhibition, susteined attention and executive function: Constructing a unifying theory of ADHD. Psychological Bulletin 121, 65-94.

Beneventi, H., Tønnessen, F. E., Ersland, L., \& Hugdahl, K. (2010). Working memory deficit in dyslexia: behavioral and fMRI evidence. International Journal of Neuroscience January 120(1), 51-59. 
Capovilla, A. G. S., \& Dias, N. M. (2008). Desenvolvimento de habilidades atencionais em estudantes da $1^{a}$ à $4^{a}$ série do ensino fundamental e relação com rendimento escolar. Psicopedagogia, 25(78), 198-211.

Coutinho, G., Mattos, P., Araújo, C., \& Duschene, M. (2007). Transtorno do déficit de atenção e hiperatividade: contribuição diagnóstica de avaliação computadorizada de atenção visual. Revista Psiquiatria Clínica, 34(5), 215222.

Cuevas, M., \& Waisburg, H. (2008). Relation between mental processing andlearning disorder in school aged children at high biological risk. Journal of the International Neuropsychological Society, 14(Suppl. 2), 12-12.

Cutting, L. E., Materek, A., Cole, C. A. S., Levine, T. M., \& Mahone, E. M. (2009). Effects of fluency, oral language, and executive function on reading comprehension performance. Annals of Dyslexia, 59, 34-54.

Dias, N. M (2009). Avaliação neuropsicológica das funções executivas: Tendências desenvolvimentais e evidências de validade de instrumentos. Dissertação de Mestrado. Programa de Pós Graduação em Distúrbios do Desenvolvimento. Universidade Presbiteriana Mackenzie, São Paulo.

Dias, N. M., Trevisan, B. T., Menezes, A., Godoy, S., \& Seabra, A. G. (no prelo). Dificuldades de aprendizagem e funções executivas. In J. Montiel \& F. C. Capovilla (Orgs.), Transtornos de Aprendizagem. São Paulo: Artes Médicas. Duncan, J., Johnson, R., Swales, M., \& Frees, C. (1997). Frontal lobe deficits after head injury: unity and diversity of function. Cognitive Neuropsychology, 14(5), 713-741.

Elliot, R. (2003). Executive functions and their disorders. British Medical Bulletin, 65, 49-59.

Estévez-González, A., García-Sánchez, C., \& Barraquer-Bordas, L. (2000). Los lóbulos frontales: El cerebro ejecutivo. Revista de Neurologia, 31(6), 566577.

Fuster, J. M. (1997). The prefrontal cortex. Philadelphia, PA: Lippincott-Raven Publishers.

García-Molia, A. (2008). Aproximación histórica a las alteraciones comportamentales por lesiones del córtex prefrontal: de Phineas Gage a Luria. Revista de Neurologia, 46, 175-181. 
Gathercole, S. E., Alloway, T. P., Willis, C., \& Adams, A. (2006). Working memory in children with reading disabilities. Journal of Experimental Child Psychology, 93, 265-281.

Gazzaniga, M. S., Ivry , R. B., \& Mangun, G. R. (2006). Neurociência cognitiva: A biologia da mente. Porto Alegre, RS: Artmed.

Gil, R. (2002). Neuropsicologia. São Paulo: Editora Santos.

Goldberg, E. (2002). O cérebro executivo: lobos frontais e a mente civilizada. RJ: Imago.

Huizinga, M, Dolan, C. V., \& Molen, M. W. Van der. (2006). Age-related in executive function: Developmental trends and a latent variable analysis. Neuropsychologia, 44, 2017-2036.

Im-Bolter, N. Johnson, J., \& Pascual-Leone, J. (2006). Processing limitations in children with specific language impairment: The role of executive function. Child Development, 77(6), 1822-1841.

Jauregi, J., Arias, C., Vegas, O., Alén, F., Martinez, S., Copet, P., \& Thuilleaux, D. (2007). A neuropsychological assessment of frontal cognitive functions in Prader-Willi syndrome. Journal of Intellectual Disability Research, 51(5), 350-365.

Landa, R. J., \& Goldberg, M. C. (2005).Language, Social, and Executive Functions in High Functioning Autism: A Continuum of Performance. Journal of Autism and Developmental Disorders, 35(5), 557-573.

Lanfranchi, S., Jerman, O., Dal Pont, E., Alberti, A., \& Vianello, R. (2010). Executive function in adolescents with Down Syndrome. Journal of Intellectual Disability Research, 54(4), 308 - 319.

Lent, R. (2001). Cem bilhões de neurônios: conceitos fundamentais de neurociência. SP: Editora Atheneu.

Levav, M. (2008). A battery for screening children affected with epilepsy in clinical practice. Journal of the International Neuropsychological Society, 14(Suppl. 2), 27-28.

Lezak, M.D., Howieson, D. B. \& Loring, D. W. (2004). Neuropsychological assessment. New York, NY: Oxford University Press.

Mahone, E. M., \& Silverman, W. (2008). ADHD and executive functions: Lessons learned from research. EP Magazine, 38, 48-51. 
McLean, J. F., \& Hitch, G. J. (1999). Working memory impairments in children with specific arithmetic learning difficulties. Journal of Experimental Child Psychology, 74, 240-260.

Menezes, A. (2008). Evidências de Validade de Instrumentos para Avaliar Funções Executivas em Alunos de $5^{a}$ a $8^{a}$ série. Dissertação de Mestrado. Programa de Pós-Graduação Stricto Sensu em Psicologia. Universidade São Francisco. Itatiba, São Paulo.

Miyake, A., Friedman, N. P., Emerson, M. J., Witzki, A. H., \& Howerter, A. (2000). The unity and diversity of executive functions and their contributions to complex "frontal lobe" tasks: a latent variable analysis. Cognitive Psychology, 41, 49-100.

Muñoz-Céspedes, J. M., \& Tirapu-Ustárroz, J. (2004). Rehabilitación de lãs funciones ejecutivas. Revista de Neurologia, 38(7), 656-663.

Natale, L. L., Teodoro, M. L. M., Barreto, G. V., \& Haase, V. G. (2008). Propriedades psicométricas de tarefas para avaliar funções executivas em pré-escolares. Psicologia em Pesquisa, 2(2), 23-35.

Nigg, J. T. (2001). Is ADHD a Disinhibitory Disorder? Psychological Bulletin, 127, 571-598.

OMS. (1993). Classificação de Transtornos Mentais e de Comportamento da CID 10: Descrição e diretrizes diagnósticas. Porto Alegre: Artes Médicas.

Orsati, F. T., Schwartzman, J. S., Brunoni, D., Mecca, T., \& Macedo, E. C. (2008). Novas possibilidades na avaliação neuropsicológica dos transtornos invasivos do desenvolvimento: análise dos movimentos oculares. Avaliação Psicológica, 7(3), 281-290.

Papazian, O., Alfonso, I., \& Luzondo, R. J. (2006). Transtornos de lãs funciones ejecutivas. Revista de Neurologia, 42(supl. 3), S45-S50.

Pliszka, S. R. (2004). Neurociência para o clínico de saúde mental. Porto Alegre: Artmed.

Rebollo, M. A., \& Montiel, S. (2006). Atención y funciones ejecutivas. Revista de Neurologia, 42(Supl 2), S3-S7.

Reiter, A., Tucha, O., \& Lange, K. W. (2005). Executive functions in children with dyslexia. Dyslexia, 11(2), 116-131. 
Rojas, S., Tapia, M., Yañez, M., \& De La Cruz, J. (2008). The Neuropsychological Assessment of a Child with Prefrontal Lobes Agenesis [Resumo em anais de congresso]. Journal of the International Neuropsychological Society, 14(Suppl. 2), 56-56.

Rowe, J., Avender, A., \& Turk, V. (2006).Cognitive executive function in Down's syndrome. British Journal of Clinical Psychology, 45, 5-17

Saboya, E., Franco, C. A., \& Mattos, P. (2002). Relações entre processos cognitivos nas funções executivas. Jornal Brasileiro de Psiquiatria, 51(2), 91-100.

Saboya, E., Saraiva, D., Palmini, A., Lima, P., \& Coutinho, G. (2007). Disfunção executiva como uma medida de funcionalidade em adultos com TDAH. Jornal Brasileiro de Psiquiatria, 56(supl.1), 30-33.

Salgado, C. A., Lima, R., \& Ciasca, S. (2008). Executive functions in students with developmental dyslexia. Journal of the International Neuropsychological Society, 14(Suppl. 2), 56-57.

Steele, S. D., Minshew, N. J., Luna, B., \& Sweeney, J. A. (2007). Spatial working memory deficits in autism. Journal of Autism and Developmental Disorders, $37,605-612$.

Sternberg, R. J. (2008). Psicologia Cognitiva (4a ed). Porto Alegre: Artmed.

Strauss, E., Sherman, E. M. S., \& Spreen, O. (2006). A Compendium of Neuropsychological Tests: Administration, norms and commentary. New York: Oxford University Press.

Wolfe, M. E. (2004). Executive function processes: inhibition, working memory, planning and attention in children and youth with attention deficit hyperactivity disorder. Tese de Doutorado, Office of Graduate Studies of Texas A \& M University.

Yerys, B. E., Hepburn , S. L., Pennington, B. F., \& Rogers, S. J. (2007). Executive function in preschoolers with autism: evidence consistent with a secondary deficit. Journal of Autism and Developmental Disorders, 37, 1068-1079.

Contato: natalia_mdias@yahoo.com.br

Recebido em: 12/02/2010

Revisado em: 04/04/2010

Aceito em: 15/06/2010 